\title{
Dégénérescence maculaire liée à l'âge
}

$\mathbf{L}$ es progrès de notre connaissance de la dégénérescence maculaire liée à l'âge (DMLA) ont concerné au premier chef les aspects cliniques de l'affection: identification plus précise des précurseurs, recherche des facteurs de risque d'apparition de la maladie par des larges études épidémiologiques, classification rigoureuse des différentes formes cliniques et de leur évolution spontanée. Surtout, la recherche récente sur la DMLA a été marquée par le développement de nouvelles stratégies conduisant à des applications thérapeutiques. La thérapeutique peut concerner au mieux la prévention de l'apparition des complications maculaires ou plus tardivement le traitement de ces complications elles-mêmes, atrophiques ou néovasculaires.

\section{La prévention}

des complications maculaires

Les molécules anti-oxydantes ont été proposées pour combattre les effets nocifs du processus oxydatif, en particulier pour piéger les radicaux libres. Les radicaux libres, en effet, dénaturent les phospholipides constitutifs des membranes des photorécepteurs qui ne peuvent être dégradés par des enzymes lysosomiales. L'altération de la phagocytose entraîne l'accumulation de déchets dans les cellules de l'épithélium pigmentaire.

Les vitamines et minéraux connus pour leur potentiel anti-oxydant sont les vitamines $\mathrm{E}$ et $\mathrm{C}$, les caroténoïdes, le sélénium, le zinc ainsi qu'un nombre d'enzymes intervenant dans les processus oxydatifs [1]. L'identification des produits d'oxydation des deux principaux pigments maculaires chez l'homme et le singe, la lutéine et la zéaxanthine [2], ainsi que l'identi- les pigments maculaires [3], a suggéré que les caroténoïdes pourraient avoir un rôle photoprotecteur contre la progression de la DMLA. Aucune des études entreprises n'a toutefois apporté la preuve d'une efficacité de l'apport supplémentaire oral en antioxydants ou en zinc.

Les drusen séreux sont considérés comme des "précurseurs", à haut risque de développement de la néovascularisation choroïdienne. Ainsi, une étude récente a démontré que le deuxième oil a un risque de survenue de néovaisseaux choroïdiens de $30 \%$, lorsqu'il présente des drusen séreux et de $60 \%$, lorsque des migrations pigmentaires s'y associent [4].

Un traitement par photocoagulation au laser dans la région maculaire peut entraîner la disparition des drusen, ce qui est connu depuis fort longtemps. Néanmoins, l'élargissement des cicatrices de laser avec le temps, a été décrit non seulement chez les myopes mais aussi après tout type de photocoagulation et en particulier, d'œdème maculaire cystoïde en grille [5]. De plus, la disparition spontanée des drusen séreux survient aussi dans $10 \%$ à 13,9\% des yeux sur une période de 5 ans. Cette disparition spontanée est plus fréquente chez les patients plus âgés (âge moyen 79 ans).

Mais surtout, il n'y a pas de preuve que la disparition des drusen séreux modifie l'évolution vers l'apparition des néovaisseaux choroïdiens et donc l'évolution spontanée de la DMLA, même si on a observé des améliorations de l'acuité visuelle après traitement au laser des drusen.

Actuellement donc, les tentatives de traitements préventifs n'ont pas fait la preuve de leur efficacité, en tout cas selon les modalités utilisées dans les différentes études.

\section{Traitement \\ des complications maculaires : l'atrophie géographique centrale}

C'est essentiellement pour l'atrophie géographique centrale que la recherche s'oriente vers la transplantation des cellules de l'épithélium pigmentaire et/ou des photorécepteurs. L'épithélium pigmentaire est considéré comme le «point de départ» de la DMLA. La transplantation de cellules jeunes et saines de cet épithélium pourrait ralentir la progression de la maladie et prévenir la perte rapide de l'acuité visuelle (voir l'article de J.A. Sahel et al., p. 1337 de ce numéro).

Dans l'ensemble, les études de transplantation de cellules de l'épithélium pigmentaire ne fournissent pas encore de résultats fonctionnels convainquants et les recherches vont peut être s'orienter vers une transplantation en bloc de photorécepteurs et d'épithélium pigmentaire. L'équipe d'Aramant a montré la faisabilité d'une transplantation en bloc des prélèvements rétiniens fœetaux attachés à l'épithélium pigmentaire rétinien chez le rat RCS: les photorécepteurs ont développé des segments internes et externes et leur marquage a montré la présence de rhodopsine, d'antigène $S$ et d' $\alpha$ transducine [6].

L'implantation de microphotodiodes est une autre approche thérapeutique, en cours de développement. Cet implant rétinien doit remplacer les photorécepteurs dégénérés. En créant des stimulus actuellement artificiels à proximité des cellules ganglionnaires, une équipe américaine a pu observer une perception visuelle utile chez des patients atteints de rétinite pigmentaire [7].

Les approches thérapeutiques des formes atrophiques de la DMLA sont 
peu nombreuses et n'ont été tentées que chez un nombre restreint de patients. En dépit de l'absence de résultats fonctionnels, ces techniques (et d'autres) doivent être affinées et poursuivies pour aboutir peut-être à une thérapeutique efficace.

\section{Néovaisseaux choroïdiens}

Jusqu'à présent la photocoagulation au laser reste la seule approche thérapeutique qui ait prouvé son efficacité dans le traitement des néovaisseaux de type visible en angiographie à la fluorescéine $[8,9]$. Ces néovaisseaux visibles ne constituent que $15 \%$ à $20 \%$ de l'ensemble des lésions à l'origine d'une maculopathie exsudative.

L'angiographie au vert d'indocyanine (ICG) permet souvent d'identifier et de localiser le type le plus fréquent de néovaisseaux visibles et les néovaisseaux occultes dans un certain nombre de cas, mais les résultats de la photocoagulation fondée sur cette méthode n'ont été publiés que dans des études pilotes sur un nombre limité de malades [10]. Une étude randomisée internationale est actuellement en cours.

- La photothérapie dynamique associe l'application d'une lumière de faible intensité et non thermique, à un médicament photosensibilisant pour provoquer des effets tissulaires localisés. Ce traitement, déjà largement utilisé en thérapeutique antitumorale, montre des résultats prometteurs pour le traitement de la néovascularisation choroïdienne. Un colorant photosensibilisant est administré par voie intraveineuse et s'accumule dans le tissu cible grâce à un vecteur sélectionné. L'irradiation lumineuse du tissu au pic d'absorption du colorant confère à celui-ci un état excité par transfert d'un électron supplémentaire. Le photosensibilisant excité revient rapidement à son état basal et transfère une partie de l'énergie à d'autres molécules. Les lésions cellulaires sont provoquées par la formation de radicaux libres qui réagissent avec des protéines, des acides nucléiques et des membranes, mais le principal mode d'action de la photothérapie dynamique est une thrombose vasculaire: la lésion des cellules endothéliales déclenche une agrégation plaquettaire, puis une stase sanguine et l'agrégation d'autres éléments sanguins et, finalement, une occlusion vasculaire [11]. Cette occlusion est transitoire et impose des séances itératives de photothérapie dynamique pour obtenir une occlusion stable de la néovascularisation choroïdienne.

Les nouveaux photosensibilisants utilisés ont une meilleure absorption à des longueurs d'onde plus longues et une photosensibilité cutanée diminuée. Le photosensibilisant actuellement en cours d'étude clinique de phase III est la benzoporphyrine monoacide-A (BPD-MA). Pour augmenter l'accumulation du photosensibilisant dans le tissu cible et pour minimiser des lésions dans les tissus de voisinage, le BPD-MA est lié à une LDL (low-density lipoprotein) qui se fixe de façon préférentielle aux cellules endothéliales des néovaisseaux choroïdiens [12, 13]. En effet, les cellules endothéliales en cours de prolifération expriment fortement des récepteurs des LDL (comme le récepteur apo $\mathrm{B} / \mathrm{E}$ ) [13]; la liaison $\mathrm{du}$ ligand entraîne l'endocytose dépendante du récepteur [14].

Bien que ce traitement soit considéré comme le plus sélectif des néovaisseaux choroïdiens, les études histologiques des néovaisseaux induits chez le singe ont mis en évidence après photothérapie dynamique des lésions au niveau de l'épithélium pigmentaire rétinien et de la couche nucléaire externe [15]. Néanmoins, ce traitement semble stabiliser l'acuité visuelle des patients à long terme dans l'étude pilote initiale, malgré l'altération provoquée des cellules de l'épithélium pigmentaire [16]. Les premiers résultats d'une grande étude contrôlée internationale seront disponibles à la fin de l'année 1999.

- L'ablation chirurgicale des membranes néovasculaires, par voie endooculaire, n'a apporté jusqu'à présent que des résultats visuels décevants dans la DMLA [17], même associée à une transplantation de cellules de d'épithélium pigmentaire adultes ou embryonnaires [18]. Les meilleurs résultats annoncent $20 \%$ d'amélioration de l'acuité visuelle et $51 \%$ de stabilisation. Une grande étude randomisée est actuellement en cours aux États-Unis. Néanmoins, cette approche thérapeutique devrait être comparée à la photocoagulation subfovéale [9] ou périfovéale [8] qui ont permis d'obtenir un bénéfice fonctionnel statistiquement significatif dans des études randomisées par rapport à l'évolution spontanée.

Les rétinectomies, prééquatoriales, de $360^{\circ}$ ou arciformes au-dessus des vaisseaux temporaux supérieurs avec rotation de la rétine, ou la résection sclérale temporale supérieure mettent les cônes de l'aire maculaire au contact d'un épithélium pigmentaire sain, non néovascularisé (voir l'article de J.A. Sahel et al., p. 1337 de ce numéro). Ces techniques chirurgicales sophistiquées sont en cours d'évaluation et témoignent de la recherche d'une méthode progressivement plus satisfaisante et adéquate, mais l'approche chirurgicale reste encore grevée de complications graves (risque de prolifération rétino-vitréenne, risque de décollement de rétine, cataracte...).

- Les médications anti-angiogéniques constituent un espoir logique qui est actuellement en réelle expansion. Les études évaluant les médicaments anti-angiogéniques sont attendues avec beaucoup d'intérêt.

L'angiogenèse dépend de nombreux facteurs. D'une part, de la synthèse de cytokines pro-angiogéniques (tumor necrosis factor- $\alpha$ [TNF- $\alpha]$, interleukine-8 [IL-8]), de facteurs de croissance (basic fibroblast growth factor [b-FGF] et vascular endothélial growth factor [VEGF]), des protéines de la matrice extracellulaire (laminine, fibrinonectine, protéo-héparanesulfate) et d'autres médiateurs endogènes favorisant l'angiogenèse. D'autre part, une synthèse insuffisante des médiateurs angiostatiques endogènes, comme certaines protéines de la matrice extracellulaire (thrombospondine), des rétinoïdes, des inhibiteurs de la métalloprotéinase, du facteur plaquettaire 4 et certains facteurs de croissance, modifient l'équilibre en faveur de la prolifération [19, 20]. Néanmoins, actuellement les modèles expérimen- 
taux de néovascularisation rétinienne et irienne restent imparfaits et ne sont pas très adaptés à une néovascularisation de type choroïdien.

Les interférons $\alpha$ et $\beta$ diminuent l'expression du bFGF et l'angiogenèse induite par le VEGF dans les modèles d'ischémie coronarienne et cornéenne [21]. L'interféron $\alpha$-2a empêche la motilité et la prolifération des cellules endothéliales capillaires [22]. Le traitement par interféron $\alpha-2$ a par voie générale de la DMLA néovasculaire n'a pourtant pas fait preuve d'efficacité dans une grande étude randomisée multicentrique [23]. En effet, après 1 an de suivi, l'acuité visuelle des patients traités était plus mauvaise que celle des patients non traités. Le traitement local par interféron $\alpha-2 a$, près $d u$ pôle postérieur à l'aide d'un ballon de Lincoff modifié, pourrait apporter des résultats et, en tout cas, l'affranchissement des effets secondaires généraux [24]. Parmi les autres molécules en cours d'évaluation dans des études pilotes, on peut citer l'interféron $\beta$ par voie systémique.

La thalidomide est une molécule immunomodulatrice qui possède une action tératogène chez le primate et le lapin. C'est une molécule inhibitrice de l'angiogenèse de néovascularisation cornéenne induite par le bFGF et le VEGF chez le lapin [25]. L'angiogenèse passe par l'intervention de deux intégrines, dont l'une $(\alpha v \beta 3)$ est induite par TNF- $\alpha$ et bFGF, l'autre intégrine $\alpha v \beta 5$ est induite par le VEGF. L'intégrine $\alpha v \beta 3$ est exprimée d'une manière préférentielle dans les vaisseaux proliférants. Des anticorps ou des antagonistes de ce peptide peuvent bloquer l'angiogenèse induite par les intégrines [21]. Deux anticorps ayant une forte affinité pour $\alpha$ vß3 ont déjà été synthétisés. Les études in vivo ont montré que l'anticorps anti- $\alpha v \beta 3$ inhibe l'angiogenèse induite par les facteurs de croissance ou les tumeurs, sans effets sur des vaisseaux préexistants [27]. La thalidomide semble avoir une action inhibitrice sur ces deux molécules d'adhérence cellulaire $(\mathrm{m} / \mathrm{s} 1997$, $\left.n^{\circ} 4, p .595\right)$. Cette molécule est actuellement évaluée dans une étude clinique randomisée américaine chez des patients présentants des néovaisseaux choroïdiens dans le cadre d'une DMLA.

Identifié comme facteur de croissance responsable d'une néovascularisation rétinienne et irienne dans les modèles animaux avec ischémie rétinienne, le VEGF semble aussi jouer un rôle dans la néovascularisation choroïdienne [27]. Pour bloquer cette voie de l'angiogenèse, différents inhibiteurs de l'action du VEGF sont en cours d'évaluation : des antagonistes des récepteurs solubles du VEGF, des oligonucléotides anti-sens de VEGF, l'antagoniste de la tyrosine kinase du récepteur du VEGF.

L'angiogenèse peut également être inhibée au niveau des métalloprotéinases de la matrice extracellulaire (MMP) et des inhibiteurs tissulaires des métalloprotéinases (TIMP). Il a été démontré que les inhibiteurs des MMP bloquent l'angiogenèse in vivo [28]. TIMP-3, en particulier, peut bloquer la migration des cellules endothéliales induite par bFGF et VEGF in vitro et la néovascularisation in vivo. Certaines MMP peuvent également avoir un effet inhibiteur. L'angiostatine, un fragment du plasminogène, exerce cette action par l'intermédiaire du MMP-12 produit par des macrophages [29]. Il existe probablement un mécanisme similaire de l'endostatine, dont le précurseur est le collagène XVIII [30]. L'angiostatine est actuellement en cours d'évaluation dans une première étude clinique.

L'effet angiostatique des corticostéroïdes, s'exerçant par les inhibiteurs des métalloprotéinases, donc sur le cytosquelette, va être évalué dans les prochaines études cliniques. Les stéroïdes seront administrés par voie sous-ténonienne.

La radiothérapie a un effet antiangiogénique par son action antimitotique et anti-inflammatoire. De multiples études pilotes ont suggéré un effet bénéfique de l'irradiation [31]. Malheureusement, ces études ne sont pas comparables entre elles du fait de critères d'inclusion extrêmement différents : des néovaisseaux visibles, occultes ou l'association des deux ont été inclus. Aucune étude ne présente de groupe témoin et aucune n'est randomisée. Sur la base des effets de la radiothérapie et des études de l'évolution spontanée des néovaisseaux occultes, une étude pilote réalisée à la Clinique ophtalmologique universitaire de Créteil a donné les résultats les plus favorables de la littérature. Une étude contrôlée et randomisée, monocentrique, est actuellement en cours à Créteil.

\section{Conclusions}

La photocoagulation au laser reste jusqu'à présent le traitement de référence pour les $15 \%$ de néovaisseaux visibles. Toutes les autres alternatives thérapeutiques des néovaisseaux visibles doivent être comparées à ce standard.

Les résultats de la photothérapie dynamique sont attendus avec beaucoup d'espoir, tant pour les néovaisseaux visibles, que pour les néovaisseaux occultes qui vont être concernés dans une nouvelle évaluation. En effet, les néovaisseaux occultes, les décollements vascularisés de l'épithélium pigmentaire et l'association de néovaisseaux choroïdiens visibles dans une plage de néovaisseaux occultes sont les formes cliniques pour lesquelles nous sommes particulièrement démunis.

Les approches thérapeutiques de l'avenir pourraient tenter d'inhiber les différentes étapes de l'angiogenèse : la prolifération, la migration et la différenciation des cellules de l'endothélium choroïdien. Le développement des inhibiteurs spécifiques des facteurs angiogéniques et de leur récepteurs, ainsi que l'inhibition de la synthèse de la matrice extracellulaire pourrait empêcher la migration des cellules endothéliales. Un effet sur la concentration de facteurs inhibiteurs naturellement présent dans la rétine et la choroïde pourrait constituer une autre approche.

Les recherches de thérapeutique expérimentale dans la DMLA permettront de progresser à condition d'en respecter les points critiques qui restent le choix de la stratégie précise à utiliser, son évaluation dans un modèle animal adapté, la détermination préalable des objectifs envisagés et la réalisation d'études cliniques rigoureuses contrôlées 


\section{RÉFÉRENCES}

1. West S, Vitale S, Hallfrisch J, Munoz B, Muller D, Bressler SB, Bressler NM. Antioxidants and supplements: protective for agerelated macular degeneration. Arch Ophthalmol 1994; 112: 222-7.

2. Khachik F, Bernstein PS, Garland DL. Identification of lutein and zeaxanthin oxidation products in human and monkey retinas. Invest Ophthalmol Vis Sci 1997; 38 : 180211.

3. Bernstein PS, Balashov NA, Tsong ED, Rando RR. Retinal tubulin binds macular carotenoids. Invest Ophthalmol Vis Sci 1997; $38: 167-75$

4. Risk factors of choroidal neovascularization in the second eye of patients with juxtafoveal or subfoveal choroidal neovascularization secondary to age-related macular degeneration. Macular Photocoagulation Study Group. Arch Ophthalmol 1997; 115: 741-7.

5. Schatz H, Madeira D, McDonald R, Johnson R. Progressive enlargement of laser scars following grid laser photocoagulation for diffuse diabetic macular edema. Arch Ophthalmol 1991 ; 109: 1549-51.

6. Aramant RB, Seiler MS, Ball SL. Cografts of fetal RPE with retina - a hope for macular degeneration. Symposium Pathogenesis and Treatment of Age-Related Macular Degeneration, Baltimore, 1998.

7. Humayun MS, De Juan E, Dagnelie G, Greenberg RJ, Propst RH, Philips H. Visual perception elicited by electrical stimulation of retina in blind humans. Arch Ophthalmol 1996; 114: 40-6.

8. Coscas G, Soubrane G. Photocoagulation des néovaisseaux sous-rétiniens dans la dégénérescence maculaire sénile par laser à argon: résultats de l'étude randomisée de 60 cas. Bull Mem Soc Fr Ophthalmol 1983; 94 : 149-54.

9. Macular Photocoagulation Study Group. Laser photocoagulation of subfoveal neovascular lesions in age-related macular degeneration : results of a randomized clinical trial. Arch Ophthalmol 1991; 109 : 1220-31.

10. Guyer D, Yannuzzi L, Slakter J. Digital indocyanine green videoangiography of occult choroidal neovascularization. Ophthalmology $1994 ; 101$ : 1727-37.

11. Castellani A, Pace G, Concioli M. Photodynamin effect of hematoporphyrin on blood microcirculation. I Pathol Bacteriol 1963; 86: 99-102.

12. Kramer M, Miller JW, Michaud N, Moulton RS, Hasan T, Flotte TJ, Gragoudas ES. Liposomal benzoporphyrin derivate verteporfin photodynamic therapy. Selective treatment of choroidal neovascularization in monkeys. Ophthalmology 1996; 103: 42738 .

13. Jori G. Low density lipoprotein-liposome delivery system for tumor photosensitizers

$\mathrm{m} / \mathrm{s} n^{\circ} 12$, vol. 14 , décembre 98 in vivo. In: Henderson B, Dougherty T, ed. Photodynamic therapy, basic principles and clinical applications. New York: Dekker, 1992: 173-86.

14. Kessel D. Porphyrin-lipoprotein association as a factor in porphyrin localization. Can Lett 1986; 33 : 183-8.

15. Miller JW, Walsh AW, Kramer M, Hasan T, Michaud N, Flotte TJ, Haimovici R, Gragoudas ES. Photodynamic therapy of experimental choroidal neovascularization using lipoprotein-delivered benzoporphyrin. Arch Ophthalmol 1995; 113: 810-8.

16. Schmidt-Erfurth U, Miller JW, Sickenberg M, Bressler NM, Laqua H, Gragoudas ES, Zografos L, Hager A, Piguet B, Birngruber R, van den Bergh H, Strong HA, Fsadni M. Photodynamic therapy for choroidal neovascularizationin a phase I/II study: preliminary results of multiple treatments. Invest Ophthalmol Vis Sci 1997; 38 (suppl) : 74.

17. Thomas MA, Dickinson JD, Melberg NS, Ibanez HE, Dhaliwal RS. Visual results after surgical removal of subfoveal choroidal neovascular membranes. Ophthalmology 1994; 101: 1384-96.

18. Algvere PV, Berglin L, Gouras P, Sheng Y. Transplantation of fetal retinal pigment epithelium in age-related macular degeneration with subfoveal neovascularization. Graefe's Arch Clin Exp Ophthalmol 1994; 232: 707-16.

19. Klagsbrun M, D'Amore PA. Vascular endothelial growth factor and its receptors. Cytokine Growth Factor Rev 1996; 7 : 259-70.

20. Polverini PJ. Inhibitors of neovascularization: critical mediators in the coordinate regulation of angiogenesis. In : Angiogenesismolecular biology, clinical aspects. New York: Plenum Press, 1989; 263: 29.

21. Tobe T, Takahashi K, Ohkuma H, Uyama. The effect of interferon-beta on experimental choroidal neovascularization. Nippon Ganka Gakkai Zasshi 1995; 99: 57181 .

22. Friesel R, Komoriya A, Maciag T. Inhibition of endothelial cell proliferation by gamma interferon. J Cell Biol 1987; 104: 689-96.

23. Pharmacological Therapy for Macular Degeneration Study Group. Interferon alfa$2 \mathrm{a}$ is ineffective for patients with choroidal neovascularization secondary to age related macular degeneration. Results of a prospective randomized placebo-controlled clinical trial? Arch Ophthalmol 1997; 115 : 865-72.

24. Lincoff H, Kreissig I, Gelisken F, Stanga $P$ A retained catheter for retrobulbar administration of interferon for age-related macular degeneration. In: Wiedemann P, Kohen L, eds. Macular and retinal diseases. Dev Ophthalmol. Basel: Karger, 1997; 29: 69-77.

25. Friedlander M, Brooks PC, Shaffer RW, Kincaid CM, Varner JA, Cheresh DA. Definition of two angiogenic pathways by dis- tinct alpha v integrins. Science 1995; 270 : 1500-2.

26. Senger DR, Claffey KP, Benes JE, Peruzzi CA, Sergiou AP, Detmar M. Angiogenesis promoted by vascular endothelial growth factor: regulation through alphalbetal and alpha2betal integrins. Proc Natl Acad Sci USA 1997; 94 : 13612-7.

27. Lopez PF, Sippy BD, Lambert HM, Thach AB, Hinton DR. Transdifferentiated retinal pigment epithelial cells are immunoreactive for vascular endothelial growth factor in surgically excised age-related macular degeneration-related choroidal neovascular membranes. Invest Ophthalmol Vis Sci 1996; $37: 855-68$

28. Gingras D, Béliveau R. L'angiogenèse tumorale : une nouvelle cible thérapeutique anticancéreuse. Med Sci 1997; 13: 1428-35.

29. Soloway P. Mechanisms involved in ECM turnover. In: Pathogenesis and treatment of age-related macular degeneration: current knowledge leads to future research. Baltimore, June 1998.

30. O'Reilly MS, Boehm T, Shing Y, Fukai N, Vasios G, Lane WS, Flynn E, Birkhead JR, Olsen BR, Folkman J. Endostatin. An endogenous inhibitor of angiogenesis and tumor growth. Cell 1997; 88: 277-85.

31. Finger PT, Berson A, Sherr D, Riley R, Balkin RA, Bosworth JL. Radiation therapy for subretinal neovascularization. Ophthalmology 1996; 103: 878-89.

\section{Gisèle Soubrane}

Professeur des universités, praticien hospitalier.

\section{Dagmar Kuhn}

Chef de clinique assistant.

\section{Gabriel Coscas}

Professeur des universités, praticien hospitalier.

Clinique ophtalmologique universitaire de Créteil, 40, avenue de Verdun, 94010 Créteil, France.

\section{TIRÉS À PART}

\section{G. Soubrane.}

